Bioelectrochemistry and Bioenergetics, 28 (1992) 247-267

A section of J. Electroanal. Chem., and constituting Vol. 343 (1991)

Elsevier Sequoia S.A., Lausanne

JEC BB 01516

\title{
Membrane electroporation and direct gene transfer *
}

\author{
Eberhard Neumann \\ Faculty of Chemistry, University of Bielefeld, PO Box 100131, D-4800 Bielefeld 1 (Germany)
}

(Received 31 December 1991)

\begin{abstract}
The direct transfer of genetic material into cells by electroporation can be described in physicochemical terms as an electroporation-resealing hysteresis. The hysteresis concept includes unidirectional state transitions of the membrane, coupled to electrodiffusive migration of DNA through cell wall structures and electroporated plasma membranes. Deeper insight into electroporation phenomena such as electrotransfection, electrofusion and electro-insertion is gained by the inspection of the electrosensitivity and the recovery curves of cell populations as well as by the analysis of the pulse strength-duration relationship. A theoretical framework is developed for an adequate comparison of data obtained with different pulse shapes. The results of the physicochemical analysis of clectroporation data not only indicate possible molecular mechanisms but are also instrumental in developing a goal-directed optimization strategy for the various practical applications of electroporation techniques such as electric gene delivery, production of hybridoma cells for antibody secretion or the insertion of immune proteins into the membranes of blood organelles.
\end{abstract}

\section{INTRODUCTION}

The electric manipulation of biological cells and cell tissue has become a widely used method in biophysics and cell biology, in particular molecular genetics, as well as in biotechnology and medicine [1-3]. The electric field strengths $E$ and pulse durations $\Delta t$ which are applied in the various electroporation methods have to be adjusted according to the cell size, the cell type and the suspension medium. Essential for the choice of the electrical parameters is the presence of cell walls or extracellular matrix structures and of intracellular submembrane filament organizations. The parameters are in the ranges $E=0.2-30 \mathrm{kV} \mathrm{cm}^{-1}$ and $\Delta t=0.01-30$ ms.

"Dedicated to Professor Giulio Milazzo on the occasion of his 80th birthday.

0302-4598/92/\$05.00 (C) 1992 - Elsevier Sequoia S.A. All rights reserved 
When the conditions for the pulse characteristics and the medium, in particular the ionic strength and the temperature, are properly chosen, electroporation causes transient and reversible (non-destructive) permeability changes in the pole cap regions of the cell surface membranes [4-7]. This electropermeabilization can be used for the artifical release of cell components [4] as well as for the uptake of foreign substances [8,9]. A further important consequence of electroporation is that electroporated membranes are also conditioned for fusion if brought into contact. The electrofusion of cells and cell organelles is a well-established electroporation method [9-14]. A more recent application of the electroporation technique is the electro-insertion of foreign glycoproteins into the membrane of blood organelles [15].

Of general interest for molecular biology, gene engineering and gene therapy is the direct electroporative transfer of DNA [16], or other nucleic acids and proteins [17], into recipient cells, micro-organisms and tissue. The main advantage of electroporative gene transfer is that intact chemically untreated cell material can be efficiently transfected [18-20]. The stable electrotransformation of intact bacteria, yeast and plant cells, in particular, is of great biotechnological potential $[1,3,21]$.

Despite the numerous applications of electroporation, the molecular mechanisms operative in the various electroporation phenomena are not well understood. Therefore data analysis and technical optimization strategies are still generally empirical. Quantitative model calculations only cover partial aspects such as electric properties [1-3]. No doubt, further progress in goal-directed applications of electroporation methods in cell biology, biotechnology and medicine will greatly benefit from understanding of the molecular mechanism of membrane electroporation.

General practical guidelines for gene delivery have already been outlined in the first documentation of electroporative gene transfer leading to cell transformation $[16,22]$. Detailed technical prescriptions for many special cases are also available [23].

In this discussion of gene transfer by electroporation, the fundamental physicochemical principles of electric field effects on cells in general, and on membranes and DNA as polyelcctrolyte structures in particular, are cmphasized. Deeper insight can be gained by inspection and analysis of strength-duration curves, the electrosensitivity of the cells and the transformation efficiencies as a function of the concentrations of cells and DNA. The various concepts and suggestions for goal-directed optimization strategies are outlined primarily with respect to very original work related to particular aspects of cell electrotransformations. A historical survey of the development and key observations of electric field effects on cells is given in ref. 24 .

\section{HYSTERESIS AND CHEMICAL ELECTROPORATION SCHEME}

Membrane electroporation is a new field of biophysical chemistry. There is still a need to classify the observations in terms of physical concepts and to establish an 


\section{TABLE 1}

Fundamental processes of membrane electroporation

\begin{tabular}{|c|c|}
\hline Physicochemical process & Electric term \\
\hline \multicolumn{2}{|l|}{ Reversible primary processes } \\
\hline \multicolumn{2}{|l|}{ Primary electric events } \\
\hline Electric dipole induction and dipole orientation & Dielectric polarization \\
\hline $\begin{array}{l}\text { Redistribution of mobile ions at membrane-solution phase } \\
\text { boundaries including } \\
\text { (a) ionic atmosphere shifts and (b) local activity changes } \\
\text { of effectors }\left(\mathrm{H}^{+} \text {or } \mathrm{Ca}^{2+} \text { ions) }\right.\end{array}$ & $\begin{array}{l}\text { Ionic interfacial } \\
\text { polarization (Maxwell- } \\
\text { Wagner, } \beta \text { dispersion) }\end{array}$ \\
\hline \multicolumn{2}{|l|}{ Structural rearrangements } \\
\hline \multicolumn{2}{|l|}{ Conformational changes in protein and lipid molecules } \\
\hline $\begin{array}{l}\text { Phase transitions in lipid domains resulting in pores, } \\
\text { cracks (via pore coalescence) and percolation }\end{array}$ & $\begin{array}{l}\text { Electroporation } \\
\text { Electropores, electrocracks, } \\
\text { electropercolation }\end{array}$ \\
\hline \multicolumn{2}{|l|}{ Annealing and resealing processes (pore closure) } \\
\hline Energy transfer by conformational cycles & $\begin{array}{l}\text { Electroconformational } \\
\text { coupling }\end{array}$ \\
\hline Non-equilibrium distribution of metabolites & Electropumping \\
\hline \multicolumn{2}{|l|}{ Irreversible secondary processes } \\
\hline Transient material exchange & Electropermeabilization \\
\hline Release of cellular compounds, e.g. haemolysis, or pDNA & Electrorelease, electrocuring \\
\hline $\begin{array}{l}\text { Uptake of external material, } \\
\text { e.g. drugs, proteins (antibodies) }\end{array}$ & Electroincorporation \\
\hline $\begin{array}{l}\text { Transfer of genetic material, e.g. } \\
\text { DNA, mRNA, viroids, with stable } \\
\text { cell transformation (insertion of } \\
\text { foreign genes into the host genome) }\end{array}$ & $\begin{array}{l}\text { Electrotransfection, } \\
\text { electrotransformation, } \\
\text { electroporative } \\
\text { gene transfer }\end{array}$ \\
\hline Cell growth and proliferation & Electrostimulation \\
\hline \multicolumn{2}{|l|}{ Membrane reorganization } \\
\hline Cell fusion (if membranes in contact) & Electrofusion \\
\hline Vesicle formation (budding) & $\begin{array}{l}\text { Electrovesiculation, } \\
\text { electrobudding }\end{array}$ \\
\hline Electromechanical rupture & Dielectric breakdown \\
\hline Insertion of proteins into membranes & Electro-insertion \\
\hline \multicolumn{2}{|l|}{ Tertiary effects } \\
\hline Temperature increase due to dissipative processes & $\begin{array}{l}\text { Joule heating, } \\
\text { dielectric losses }\end{array}$ \\
\hline Metal ion release from metal electrodes & Electro-injection \\
\hline Electrode surface $\mathrm{H}$ and $\mathrm{O}$ (nascent) & Electrolysis \\
\hline
\end{tabular}

Modified version of tables appearing in refs. 24 and 25.

unequivocal physicochemical terminology. So far, all electroporation data indicate that reversible primary processes and irreversible secondary events can be differentiated (Table 1). 


\section{Electroporation hysteresis}

Transient membrane electroporation can be viewed as a cycle of electrochemical membrane events. In the presence of an external field $E$ there is initiation of pore formation (path $\mathrm{O} \rightarrow \mathrm{A}$ ) and at pulse termination $(E=0)$ there is initiation of pore resealing (path $\mathrm{B} \rightarrow \mathrm{O}$ ) (Fig. 1). The state cycle $\mathrm{O} \rightarrow \mathrm{A} \rightarrow \mathrm{B} \rightarrow \mathrm{O}$ thus represents a relaxation hysteresis [24,25]. Crater formation [24] as evidenced by electron microscopy [26] can be classed as a field effect also involving osmotic processes.

The structural changes associated with electroporation hysteresis are most generally described by the degree of poration $\xi_{\mathrm{p}}$. The contributions $\tilde{G}(r, E)$ of the free enthalpy, i.e. the (reversible) work of electric interfacial polarization and of the structural changes, can be expressed in terms of the mean pore radius $r$ and the external electric field strength $E$. Figure 1 shows the cycle of changes in $\tilde{G}(r, E)$ associated with the hysteresis in $\xi(E)$.

The electroporation model proposed by Chizmadzhev and co-workers $[27,28]$ assumes that during pore formation there is a structural transition from hydrophobic (HO) to hydrophilic (HI) pores [29-31] when a critical pore radius $r_{c}$ is
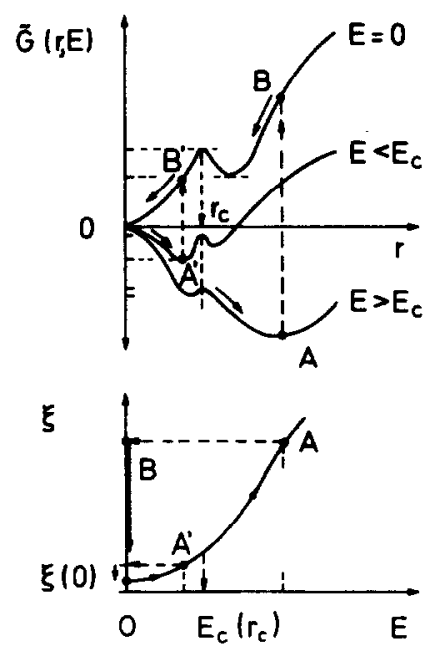

Fig. 1. The thermodynamics of electroporation hysteresis. The upper part shows the free enthalpy $\tilde{G}(r, E)$ associated with the electric and surface work as a function of pore radius $r$. The lower part shows the corresponding cycle of pore formation and resealing in terms of the degrec of poration $\xi_{\mathrm{P}}$ as a function of field strength. In the model of Chizmadzhev and co-workers [27] the changes in the structural membrane involve a transition from hydrophobic to hydrophilic pores at a critical pore radius $r_{\mathrm{c}}$, which corresponds to a critical degree of poration $\xi_{\mathrm{p}, \mathrm{c}}$ which is reached at a critical field $E_{\mathrm{c}}$. The majority of the electroporation phenomena appear to require that $E \geq E_{\mathrm{c}}$. Only the electro-insertion of glycoproteins in organelle membranes appears to be on the low field level of increased hydrophobic pores, i.e. at $E \leq E_{\mathrm{c}}$. The sequence of state transitions $\mathrm{O} \rightarrow \mathrm{A} \rightarrow \mathrm{B} \rightarrow \mathrm{O}$ underlying a supercritical $\left(E \geq E_{\mathrm{c}}\right)$ electroporation-resealing cycle is indicated. 
reached. As seen in Fig. 1, $r_{\mathrm{c}}$ corresponds to a critical degree of poration $\xi_{\mathrm{P}, \mathrm{c}}$ on the lower hysteresis branch. However, it is the detailed molecular rearrangements of the lipids in the porous membrane which are not yet known.

\section{Chemical electroporation scheme}

The various electroporation phenomena can be described by a general chemical scheme [32,33]:

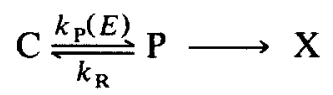

In eqn. (1), $C$ is the initial membrane state in the absence of external fields. The states $P$ represent a collection of permeabilized (and fusogenic) states of various longevities; $k_{\mathrm{P}}(E)$ and $k_{\mathrm{R}}$ are the (overall) rate coefficients of electroporation and resealing respectively. If the electroporation hysteresis is coupled to other processes such as material release or uptake, cell lysis, membrane fusion contacts or protein insertion, the states $\mathrm{X}$ have to be explicitly specified.

The hysteretic nature of the permeabilization-resealing cycle justifies treating the individual rearrangements $\mathrm{C} \rightarrow \mathrm{P}, \mathrm{C} \leftarrow \mathrm{P}$ and $\mathrm{P} \rightarrow \mathrm{X}$ as unidirectional (irreversible) processes. Usually, the reverse transitions $C \leftarrow P$ and some $P \rightarrow X$ processes occur at $E=0$; thus they represent after-pulse effects. It appears that the transport of DNA through the electroporated membrane can also involve an after-pulse phase of purely diffusive character $[16,24]$.

\section{ELECTROSENSITIVITY OF CELL POPULATIONS}

The electrotransformation of cells, bacteria and other micro-organisms is preferably performed at low, but finite, electrical conductivity. The field effect on the surface membrane is mediated by ionic interfacial polarization and thus requires a finite salt concentration. Joule heating and other tertiary effects (see Table 1) are reduced at low ionic strength. Joule heating alone, however, can be favourable for gene transfer [20]. The interpretation of electroporation data of media with low conductivity necessitates an extension of the analytical framework in terms of the solution conductivity $[24,32,33]$.

\section{Permeabilization and recovery}

Before the actual electrotransfection experiments are performed, the electrosensitivity of the cells is tested and survival or recovery curves are measured in the absence of added DNA [34]. The fraction $f(R)=R / C_{\mathrm{T}}$ of recovered (viable) cells $R$ or the percentage $R(\%)=10^{2} f(R)$ of the total cell number $C_{\mathrm{T}}$ is plotted as a function of the (initial) field strength at a given pulse duration $t_{P(E)}$, or $R(\%)$ is given as a function of $t_{P(E)}$ at given field strengths. Another variable determining $f(R)$ is the pulse number. The single pulses in a train of pulses are not 


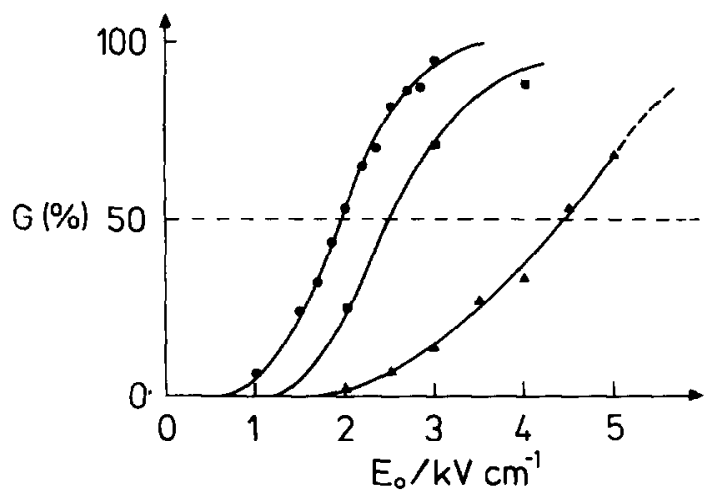

Fig. 2. Electrosensitivity $G(\%)$ of a suspension of green algae cells $C h$. reinh. (wild type 11-32c, Göttingen) to quasi-rectangular electric pulses of initial field intensity $E_{0}$ and pulse length $t_{\mathrm{p}}=0.2 \mathrm{~ms}$ at different medium conductivities: $\bullet, \lambda_{0}=(3.5 \pm 0.1) \times 10^{-4} \mathrm{~S} \mathrm{~cm}^{-1} ; \mathbf{m}, \lambda_{\mathrm{o}}=(1.5 \pm 0.1) \times 10^{-4} \mathrm{~S}$ $\mathrm{cm}^{-1} ; \Delta, \lambda_{\mathrm{o}}=(5.6 \pm 0.5) \times 10^{-5} \mathrm{~S} \mathrm{~cm}^{-1} . G(\%)$ is the percentage of cells which were critically permeabilized such that the (lethal) dye Serva Blue $\mathrm{G}\left(M_{\mathrm{r}}=854\right.$; largest dimension, $\left.2.5 \mathrm{~nm}\right)$ was taken up, thus visibly colouring these cells.

generally equivalent. Because of the longevity (seconds, minutes) of the pulse-induced structural changes in the membrane patches (pole caps) compared with the short pulse duration $(0.02-30 \mathrm{~ms})$, a second pulse encounters patches which are not yet resealed. Rotational diffusion will change the position of the cell or of an elongated micro-organism like a bacterium relative to the field vector. The analysis of electroporation effects caused by a train of pulses is thus rather complicated. In any case the $f(R)$ correlations will also depend on the composition of the electroporation medium and on the temperature.

If dye staining of the electroporated cells can be measured, as in the case of the green algae cells of the species Chlamydomonas reinhardtii (Ch. reinh.), a particularly efficient procedure can be used to find the optimum electroporation conditions for transformations or other manipulations [31,32]. The number of $P_{0}$ of permeabilized cells $P$ is determined in the presence of the staining dye during pulsing and at various times after pulse applications. The data in Fig. 2 show not only that the degree of permeabilization $f\left(P_{0}\right)$, expressed as the percentage of coloured cells $G_{0}(\%)=P_{0}(\%)=10^{2} f\left(P_{0}\right)$, is dependent on the medium conductivity, but also that Ch.reinh. cells represent a population of cells of different electrosensitivity.

The after-pulse staining procedure permits the determination not only of $P_{0}$ but also of $P_{\infty}$, the number of deadly permeabilized cells, both as a function of $E$ at a given $t_{P(E)}$ and as a function of $t_{P(E)}$ at given $E$. Figure 3 shows that the difference $\Delta G=\Delta P=P_{0}-P_{\infty}$ has a maximum in the range $\Delta E_{0}$. Thus there is a "survival optimum" where further cell manipulations can be performed with highest efficiency. The range $\Delta E_{0}$ is shifted when the electroporation conditions of ionic strength, temperature, pulse duration and pulse number are changed. Obvi- 


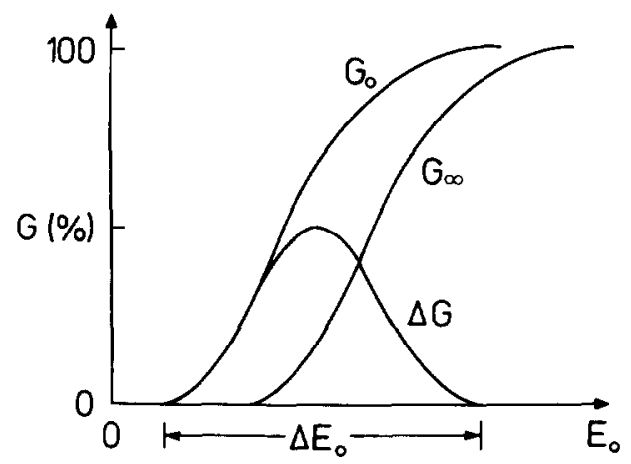

Fig. 3. Scheme for the optimization of electroporation conditions. $G_{0}=P_{0}(\%)$ is the percentage of cells coloured by the dye present during the pulse, and $G_{\infty}=P_{\infty}(\%)$ is the fraction of cells not recovered after long resealing times $[32,33]$. The difference $\Delta G-G_{0}-G_{\infty}$ represents the transiently permeabilized surviving cells. The optimum field strength range $\Delta E_{0}$ is shifted when the electroporation conditions are changed.

ously, $\Delta E_{0}$ and $\Delta P$ must be investigated for every particular cell type. In summary, the dye method originally applied as a qualitative tool to estimate the state of electroporated cells [16] can be quantified to yield useful information for an efficient theoretically guided optimization of electrical cell manipulations.

\section{Inhomogeneity of electrosensitivity}

The data in Fig. 2 confirm that biological cell populations are usually inhomogeneous in cell size, state or growth or metabolic conditions. Non-spherical cells such as bacterium rods in solution initially have different positions relative to the external electric field vector. Because of the predominantly negative surface charges of the cell wall, ionic atmosphere polarization (leading to an induced macrodipole moment) will orient the rods with their longest axis in the direction of the external electric field [35]. Both oriented bacterium rods and the oriented polyelectrolyte DNA will electrophoretically migrate with different velocities along the electric field lines. All these factors cause a distribution of the critical field strength $E_{\mathrm{c}}$ at which a cell starts either to take up a particular dye or a DNA molecule, or to fuse with other cells when brought into contact with them. The $E_{\mathrm{c}}$ values for the various cell manipulations coupled to membrane electroporation are not necessarily equal. It should be noted that the $E_{\mathrm{c}}$ term discussed in the context of Fig. 1 refers to a special structural transition.

Therefore the data in Fig. 2 strictly represent only the electrosensitivity of the Ch.reinh. cells with respect to dye uptake in terms of a distribution of critical field strengths $E_{\mathrm{c}}$. If we take the field strength $E_{0}(50 \%)$, where $G(\%)=P_{0}(\%)=10^{2}$ $f\left(P_{0}\right)=50$, as representative of the cell population, we can define a mean value

$\bar{E}_{\mathrm{c}}=E_{0}(50 \%)$ 
in terms of a Gaussian distribution [32,33]. The "width" of the electrosensitivity of a cell population can then be given in terms of a variance $\pm \Delta E_{\mathrm{c}}$. Consistent with the concept of ionic interfacial polarization preceding the membrane electroporation process, the "individual" $E_{\mathrm{c}}$ value depends on the size of the individual cell of a given population. For spherical cells, the mean value $\bar{E}_{\mathrm{c}}$ corresponds to á mean value of the cell radius $\bar{a}$ and the variance $\pm \Delta E_{\mathrm{c}}$ reflects a variance $\pm \Delta a$ in the effective cell size.

More complex cell populations may require more than one Gaussian parameter set. In any case, the definition of means like $\bar{E}_{\mathrm{c}}$ and $\bar{a}$ avoids the ambiguity encountered in the exact practical determination of a threshold field strength as the lowest $E_{\mathrm{c}}$ value of the whole population. Because of the scatter of the experimental data it is usually only possible to obtain a range $\Delta E_{\mathrm{th}}$ rather than an exact threshold value $E_{\mathrm{th}}$.

\section{Strength-duration relationship}

A further aspect of great practical significance is the field strength-pulse duration relationship, originally discussed in terms of the threshold field strength $E_{\mathrm{th}}$. Because of the uncertainties in the experimental $E_{\mathrm{th}}$ value and because of the distribution reflected in the electrosensitivity curve, the mean value $\bar{E}_{\mathrm{c}} \pm \Delta E_{\mathrm{c}}$ appears to be a more instructive parameter than just the minimum value $E_{\mathrm{th}}$ of the cell distribution.

Figure 4 shows the strength-duration relationship of Ch.reinh. cells in terms of the $\bar{E}_{\mathrm{c}}$ values for the dye penetration. Since the electroporation is initiated by

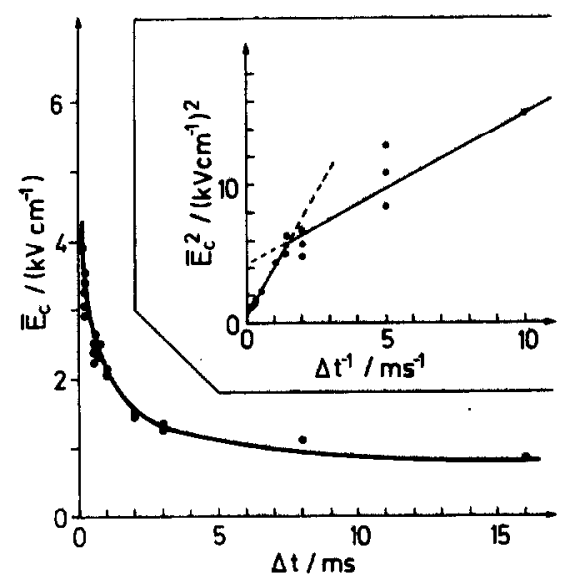

Fig. 4. Strength-duration relationships of the electroporation of $C h$. reinh. cells: mean values $\bar{E}_{\mathrm{c}}$ as a function of the pulse duration $\Delta t=t_{\mathrm{p}}$. The inset shows the data plot according to the interfacial polarization flow model: $\bar{E}_{\mathrm{c}}^{2}=A t_{\mathrm{p}}^{-1}$ where $A$ is a constant (see eqn. (19) of the text). Conditions: $\lambda_{0}=(1.4 \pm 0.4) \times 10^{-4} \mathrm{~S} \mathrm{~cm}^{-1}$ such that $f(\lambda)=1$; one rectangular pulse; $293 \mathrm{~K}$; cell density, $2 \times 10^{7} / \mathrm{ml}$. 
ionic interfacial polarization in conductive media, the energy input $W_{\mathrm{p}, \mathrm{c}}$ per cell can be expressed as

$\bar{W}_{\mathrm{p}, \mathrm{c}}=$ const. $\bar{E}_{\mathrm{c}}^{2} t_{\mathrm{p}}$

suggesting a linear relationship between $\bar{E}_{\mathrm{c}}^{2}$ and $t_{\mathrm{p}}^{-1}$ according to $\bar{E}_{\mathrm{c}}^{2}=$ $\left(\bar{W}_{\mathrm{p}, \mathrm{c}} /\right.$ const. $) t_{\mathrm{p}}^{-1}$.

For media of low conductivity and for large cells, where the relaxation time $\tau_{\mathrm{p}}$ of the interfacial polarization is of the same order of magnitude as the pulse duration $t_{\mathrm{p}}$, an effective $t_{\mathrm{p}, \text { eff }}$ must be calculated for the theoretical analysis; see also eqn. (19) below.

The insert in Fig. 4 shows that for Ch.reinh. cells there appear to be at least two electrosensitivity ranges of the type described by eqn. (3). It is not yet clear whether in the long-pulse range $\left(t_{\mathrm{p}}=\Delta t \geq 5 \mathrm{~ms}\right)$ electromechanical cell distortions [36-38] and/or osmotic effects have to be considered, or whether $\bar{E}_{\mathrm{c}}$ reaches a saturation value at large $t_{\mathrm{p}}$ which is independent of $t_{\mathrm{p}}$.

In any case, the numerical value of the critical field strength (and of the threshold field strength) is useful information only if the duration of the single pulse application is also given. If pulses can be applied for a long time or if repetitive pulses are applied in pulse trains, the threshold for an electroporation phenomenon may be very low [39].

\section{MEMBRANE POLARIZATION AND CONDUCTIVITY}

As far as the externally applied electric field $E$ is concerned, the effective membrane electroporation is an indirect field effect. The coupling of $E$ to the membrane structure is mediated by the ionic interfacial polarization. In brief, the electroporation data, inclusively those shown in Fig. 2, indicate that the field effect is a sequence [24]

$E \rightarrow \Delta \phi \rightarrow \Delta \xi$

where $E$ induces the transmembrane potential difference $\Delta \phi$ which, in turn, represents a contribution $\bar{E}_{\mathrm{m}}(\Delta \phi)$ to the mean electric field forces causing structural rearrangements $\Delta \xi$ in the membrane phase.

\section{Ionic interfacial polarization}

In the case of rectangular pulses the build-up of the induced interfacial transmembrane voltage with time $t$ is described by a linear differential equation. Integration yields

$\Delta \phi(t)=\Delta \phi(E)\left[1-\exp \left(-t / \tau_{\mathrm{p}}\right)\right]$

where $\Delta \phi(E)$ is the (time-independent) amplitude and $\tau_{\mathrm{p}}$ is the polarization time constant. 


\section{Polarization time constant}

For spherical cells of radius $a$ the value of $\tau_{\mathrm{p}}$ can be estimated from the conductivities $\lambda_{\mathrm{o}}, \lambda_{\mathrm{m}}$ and $\lambda_{\mathrm{i}}$ of the solution, the membrane and the cell interior respectively according to [40]

$\tau_{\mathrm{p}}=a C_{\mathrm{m}}\left(\lambda_{\mathrm{i}}+2 \lambda_{\mathrm{o}}\right) /\left[2 \lambda_{\mathrm{i}} \lambda_{\mathrm{o}}+\lambda_{\mathrm{m}}\left(\lambda_{\mathrm{i}}+2 \lambda_{\mathrm{o}}\right)\right]$

In eqn. (6), $C_{\mathrm{m}} \approx 1 \mu \mathrm{F} \mathrm{cm}^{-2}$ is the specific membrane capacitance and $\lambda_{\mathrm{m}}=a G_{\mathrm{m}}$ is the membrane conductivity, where $G_{\mathrm{m}}$ is the specific membrane conductance. Before the onset of transmembrane ion flow the inequality $\lambda_{\mathrm{m}} \ll \lambda_{\mathrm{o}}$ usually applies. In media of low conductivity $\left(\lambda_{\mathrm{o}} \leq 10^{-4} \mathrm{~S} \mathrm{~cm}^{-1}\right)$ we have $\lambda_{\mathrm{o}} \ll \lambda_{\mathrm{i}}$, since, at an ionic strength of $0.1 \mathrm{t}, \lambda_{\mathrm{i}} \approx 10^{-2} \mathrm{~S} \mathrm{~cm}-1$. In this case eqn. (7) takes the simple form

$\tau_{\mathrm{p}}=a C_{\mathrm{m}}\left(2 \lambda_{\mathrm{o}}\right)^{-1}$

demonstrating the inverse dependence of $\tau_{\mathrm{p}}$ on $\lambda_{\mathrm{o}}$. If larger Wien effects are absent, we can use the approximation $\tau=\tau(E=0)$ and the low field value $\lambda_{\mathrm{o}}(0)$. In many electroporation experiments we have $\tau_{p} \ll t_{p(E)}$ such that we can use the constant stationary value $\Delta \phi(E)$.

\section{Transmembrane voltage}

For spherical cells of radius $a \gg d$, where $d$ is the membrane thickness, the stationary value of the induced transmembrane potential difference is given by [24]

$\Delta \phi(E)=-\frac{3}{2} f(\lambda) a E|\cos \delta|$,

where $\delta$ is the angle between the membrane site considered and the direction of the $E$ vector; the conductivity factor $f(\lambda)$ is an explicit function of $\lambda_{o}, \lambda_{i}, \lambda_{m}$ and the ratio $d / a$. For $\lambda_{\mathrm{i}}, \lambda_{\mathrm{o}} \gg \lambda_{\mathrm{m}}, f(\lambda)=1$. It is important to note that, according to Maxwell's definition $(E=-\nabla \phi)$, the electric potential drops for positive ions in the direction of the external field vector (Fig. 5); this explains the negative sign in eqn. (8).

The membranes of biological cells have natural potential differences $\Delta \phi_{\mathrm{m}}$ due to diffusional permselectivities and $\Delta \phi_{\mathrm{s}}$ due to an asymmetric distribution of fixed surface charges. The total potential difference $\Delta \phi_{\mathrm{m}}$ experienced by a membrane component in the presence of an external field is shown in Fig. 5 [24].

At the pole caps of spherical cells, i.e. at $\delta=0^{\circ}$ and $\delta=180^{\circ}$ hence $\cos \delta= \pm 1$, the induced transmembrane voltage is a maximum. The maximum stationary value is given by

$\Delta \phi_{\text {cap }}=-\frac{3}{2} f(\lambda) E a$ 

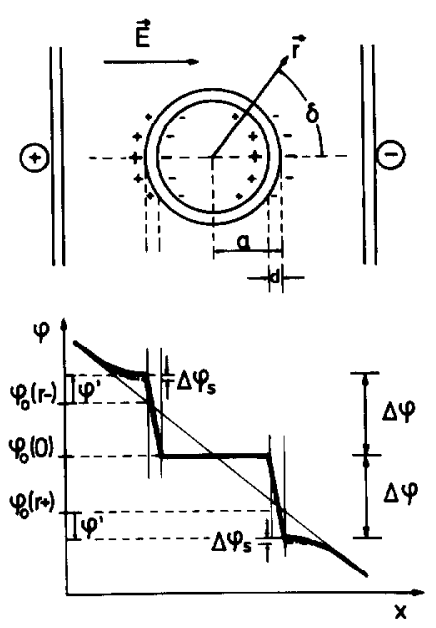

$\Delta \varphi_{M}=-\left[\frac{3}{2} f(\lambda) E_{a}+\frac{\Delta \varphi_{m}-\Delta \varphi_{5}}{\cos \delta}\right]|\cos \delta|$

Fig. 5. Interfacial polarization of a spherical non-conducting shell of thickness $d$ and outer radius $a$ in a constant external field $\boldsymbol{E}$. The stationary electric potentials are given in polar co-ordinates of the radius vector $r$ and the angle $\delta$, such that the conducting interior of the cell has the constant reference potential $\phi_{0}=0$. The $\Delta \phi$ terms are the interfacially induced transmembrane potential differences in the absence of fixed ionic groups and adsorbed ions (surface potential $\Delta \phi_{\mathrm{s}}=0$ ). The chain line schematically models the potential profile in the presence of fixed surface charges (here negative) (see text).

\section{Bacterium cells}

If cell geometries other than spheres have to be considered, we can use ellipsoidal bodies in terms of half-axes $a_{j}$ [41]. For each half-axis $a_{j}$ we can write a (modified) Fricke equation for the stationary value in the direction of $a_{j}$ as

$\Delta \phi_{j}=-F_{j} f_{j}(\lambda) a_{j} E|\cos \delta|$

where $F_{j}$ is the form factor. For spheres, $F_{j}=F=1.5$ and $a_{j}=a$, the radius.

In the case of cylindrical bacteria of length $L$ and thickness $b$ we can apply the prolate limit $L \gg b$. For bacterium rods oriented with their long axes $\left(a_{\mathrm{L}}=L / 2\right)$ in the direction of $E$, the form factor is approximated by $F_{\mathrm{L}}=1.0$ [41]. Therefore at the cap regions

$\Delta \phi_{\text {cap }}(L)=-\frac{1}{2} L E$

For Escherichia coli bacteria with an average rod length $L=3.5 \mu \mathrm{m}$ and rectangular pulses with $t_{\mathrm{p}}=1 \mathrm{~ms}$, a mean value $\bar{E}_{\mathrm{c}}=2 \mathrm{kV} \mathrm{cm}-1$ can be estimated from the data of ref. 39. Inserting this $\bar{E}_{\mathrm{c}}$ value in eqn. (11) yields $\Delta \bar{\phi}=-0.35 \mathrm{~V}$ (for $t_{\mathrm{p}}=1$ $\mathrm{ms}$ ) as the critical transmembrane potential drop at which oriented bacteria of 
mean size start to take up DNA. For longer pulse durations and multiple pulsing, $|\Delta \bar{\phi}|$ and thus $\bar{E}_{\mathrm{c}}$ may have much smaller values. In any case, $|\Delta \phi|=0.35 \mathrm{~V}$ is within the order of magnitude $0.2-0.4 \mathrm{~V}$ expected for long pulses. It is recalled that for short pulses $\left(t_{\mathrm{p}} \approx 0.01-0.1 \mathrm{~ms}\right),|\Delta \phi| \approx 1 \mathrm{~V}[42]$.

\section{Membrane conductivity}

Data of the type plotted in Fig. 2 can be used to determine the conductivity $\lambda_{\mathrm{m}}$ of the membrane, including cell wall or other envelope structures [31,32]. If the inequality $\lambda_{\mathrm{m}} \ll \lambda_{\mathrm{i}}, \lambda_{\mathrm{o}}$ holds, the $f(\lambda)$ factor of spherical cells is given by [24]

$f(\lambda)=\frac{\lambda_{\mathrm{o}} \lambda_{\mathrm{i}} 2 d / a}{\left(2 \lambda_{\mathrm{o}}+\lambda_{\mathrm{i}}\right) \lambda_{\mathrm{m}}+(2 d / a) \lambda_{\mathrm{o}} \lambda_{\mathrm{i}}}$

For media of low conductivity $\left(\lambda_{\mathrm{o}} \ll \lambda_{\mathrm{i}}\right)$ eqn. (12) reduces to

$f(\lambda)^{-1}=1+\left(\lambda_{\mathrm{m}} a / 2 d\right) \lambda_{\mathrm{o}}^{-1}$

Insertion of eqn. (13) into eqn. (9) leads to an expression relating $\bar{E}_{\mathrm{c}}$ to $\lambda_{\mathrm{o}}^{-1}$ by

$\bar{E}_{\mathrm{c}}=-\frac{2 \Delta \phi_{\mathrm{c}}}{3 \bar{a}}\left(1+\frac{\lambda_{\mathrm{m}} \bar{a}}{2 d \lambda_{\mathrm{o}}}\right)$

Equation (14) suggests a simple evaluation of the minimum value of $\left(\bar{E}_{\mathrm{c}}\right)_{0}=$ $-2 \Delta \bar{\phi}_{\mathrm{c}} / 3 \bar{a}$, and thus of $\Delta \bar{\phi}_{\mathrm{c}}$ and the membrane conductivity $\lambda_{\mathrm{m}}$, from the slope of the graphical data plot as in Fig. 6. The electroporation data of Ch.reinh. cells yield $\vec{E}_{\mathrm{c}}(f=1)=1.7 \mathrm{kV} \mathrm{cm}^{-1}$ and $\left(\lambda_{\mathrm{o}}^{-1}\right)_{0}=-1.3 \times 10^{4} \mathrm{~S}^{-1} \mathrm{~cm}$. The geometrical "radius" of the most abundant cells is $\bar{a}=3.5 \mu \mathrm{m}$ and the membrane thickness is taken to be $d=10^{-6} \mathrm{~cm}$. Insertion yields $\Delta \bar{\phi}_{\mathrm{c}}=-0.9 \mathrm{~V}$ and $\lambda_{\mathrm{m}}=4.4 \times 10^{-7} \mathrm{~S}$ $\mathrm{cm}^{-1}$.

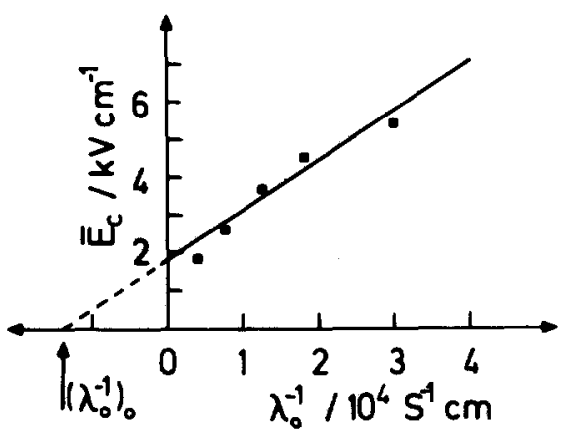

Fig. 6. The dependence of the mean critical field strength $\bar{E}_{\mathrm{c}}$ for dye uptake by electroporation of $\mathrm{Ch}$. reinh. cells on the solution conductivity $\lambda_{\mathrm{o}}$ at $293 \mathrm{~K}$ according to eqn. (14) (see the legend to Fig. 2). Data evaluation yields the mean critical transmembrane voltage $\Delta \bar{\phi}_{\mathrm{c}}=-0.9 \mathrm{~V}$ and the membrane conductivity $\lambda_{\mathrm{m}}=4.4 \times 10^{-7} \mathrm{~S} \mathrm{~cm}^{-1}$. 
The dependence of $\bar{E}_{\mathrm{c}}$ on $\lambda_{\mathrm{o}}$ (Fig. 6) is quantitatively consistent with the notion of an indirect field effect on the membrane structure: electropore formation is mediated by interfacial polarization. Further, at low solution conductivity we cannot neglect $\lambda_{\mathrm{m}}$; instead of $f(\lambda)=1$, eqn. (13) must be applied.

\section{PULSE SHAPE AND DATA COMPARISON}

Frequently, electroporation data which were obtained using different pulse shapes are compared. However, an adequate comparison requires a deeper insight into the details of the membrane polarization process.

The ion accumulations at the membrane interfaces, primarily at the pole caps (Fig. 5), can be described as resulting from an interfacial "polarization current" $I_{\mathrm{p}}$ and a corresponding surface "polarization conductance" $G_{\mathrm{p}}$. The electric part $W_{\mathrm{p}}$ of the free enthalpy change or reversible work for the induction of the transmembrane voltage $\Delta \phi$ and the subsequent pore formation is then expressed as

$W_{\mathrm{p}}=\int_{0}^{t} I_{\mathrm{p}}|\Delta \phi| \mathrm{d} t$

In the ohmic region, where $G_{\mathrm{p}}$ in $I_{\mathrm{p}}=G_{\mathrm{p}}(-\Delta \phi)$ is a constant, we obtain

$W_{\mathrm{p}}=G_{\mathrm{p}} \int_{0}^{t}(\Delta \phi)^{2} \mathrm{~d} t$

\section{Rectangular pulses}

If the pulse duration $t_{\mathrm{p}}$ and the polarization time constant $\tau_{\mathrm{p}}$ are comparable, $W_{\mathrm{p}}$ is a function of both $\tau_{\mathrm{p}}$ and $t_{\mathrm{p}}$. Substitution of eqn. (5) into eqn. (16) and integration between the limits $t=0$ and $t=t_{\mathrm{p}}$ yields

$W_{\mathrm{p}}=G_{\mathrm{p}}[\Delta \phi(E)]^{2} t_{\mathrm{p}, \mathrm{eff}}$

where the effective pulse duration is given by

$t_{\mathrm{p}, \text { eff }}=t_{\mathrm{p}}-\frac{\tau_{\mathrm{p}}}{2}\left[3+\exp \left(-\frac{2 t_{\mathrm{p}}}{\tau_{\mathrm{p}}}\right)-4 \exp \left(-\frac{t_{\mathrm{p}}}{\tau_{\mathrm{p}}}\right)\right]$

Cell size and ionic content are often such that $\tau_{\mathrm{p}} \ll t_{\mathrm{p}}$. In this limit, $t_{\mathrm{p}, \text { eff }}=t_{\mathrm{p}}$ and $W_{\mathrm{p}}=G_{\mathrm{p}}[\Delta \phi(E)]^{2} t_{\mathrm{p}}$

At the cap regions of spherical cells, where cqn. (9) is valid, the mean polarization energy $\bar{W}_{\mathrm{p}, \mathrm{c}}$ of a cell distribution $\bar{a} \pm \Delta a$ is given by

$\bar{W}_{\mathrm{p}, \mathrm{c}}=G_{\mathrm{p}}\left[\frac{3 \bar{a}}{2} f(\lambda)\right]^{2} \bar{E}_{\mathrm{c}}^{2} t_{\mathrm{p}}$

thus specifying the constant in eqn. (3) in more detail. 
Condenser discharge pulses

The exact analysis of cxponential pulse intensities is more elaborate. Condenser discharge pulses of the form

$E(t)=E_{0} \exp \left(-t / \tau_{\mathrm{E}}\right)$

where $\tau_{\mathrm{E}}=R^{\prime} C^{\prime}$ and $R^{\prime}$ and $C^{\prime}$ are the resistance and the capacitance respectively of the discharge circuit [24], induce a transmembrane voltage of

$\Delta \phi(t)=\Delta \phi\left(E_{0}\right) \frac{\tau_{\mathrm{E}}}{\tau_{\mathrm{E}}-\tau_{\mathrm{p}}}\left[\exp \left(-\frac{t}{\tau_{\mathrm{E}}}\right)-\exp \left(-\frac{t}{\tau_{\mathrm{p}}}\right)\right]$

If $\tau_{\mathrm{p}}$ is field independent (see ref. 24 for details), substitution of eqn. (21) into eqn. (16) yields

$W_{\mathrm{p}}=G_{\mathrm{p}}\left[\Delta \phi\left(E_{0}\right)^{2}\left(\frac{\tau_{\mathrm{E}}}{\tau_{\mathrm{E}}-\tau_{\mathrm{p}}}\right)^{2} X\right]$

where

$$
\begin{aligned}
X= & -\frac{\tau_{\mathrm{E}}}{2}\left[\exp \left(-\frac{2 t}{\tau_{\mathrm{E}}}\right)-1\right]-\frac{\tau_{\mathrm{p}}}{2}\left[\exp \left(-\frac{2 t}{\tau_{\mathrm{p}}}\right)-1\right] \\
& +\frac{2 \tau_{\mathrm{E}} \tau_{\mathrm{p}}}{\tau_{\mathrm{E}}-\tau_{\mathrm{p}}}\left\{\exp \left[-\frac{t\left(\tau_{\mathrm{E}}+\tau_{\mathrm{p}}\right)}{\tau_{\mathrm{E}} \tau_{\mathrm{p}}}\right]-1\right\}
\end{aligned}
$$

Quasi-rectangular pulses

For quasi-rectangular (QR) pulses the upper integration boundary is $t=t_{\mathrm{p}}$. In the case of very rapid membrane polarization $\left(\tau_{p} \ll \tau_{E}\right)$ we obtain from eqns. (21)-(23)

$\Delta \phi(t)=\Delta \phi\left(E_{0}\right) \exp \left(-t / \tau_{\mathrm{E}}\right)$

and

$W_{\mathrm{p}}=G_{\mathrm{p}}\left[\Delta \phi\left(E_{0}\right)\right]^{2} \frac{\tau_{\mathrm{E}}}{2}\left[1-\exp \left(-\frac{2 t}{\tau_{\mathrm{E}}}\right)\right]$

For QR pulses we take $t=t_{\mathrm{p}}$.

Purely exponential pulses

In the case of purely exponential (CD) pulses the limit $t \rightarrow \infty$ applies and the polarization energy is given by

$$
W_{\mathrm{p}}=G_{\mathrm{p}}\left[\Delta \phi\left(E_{0}\right)\right]^{2} \frac{\tau_{\mathrm{E}}}{2}
$$




\section{Data comparison}

An adequate comparison of electroporation data can now be based on the condition of equal polarization energy. For instance, we compare data obtained from applications of rectangular pulses and CD pulses, for the conditions $\tau_{\mathrm{p}} \ll t_{\mathrm{p}}$ and $\tau_{p} \ll \tau_{E}$ respectively. Hence eqns. (19) and (26) apply and for the mean values of the cell population we obtain

$\bar{E}_{\mathrm{c}}^{2} t_{\mathrm{p}}=\left(\bar{E}_{0}\right)_{\mathrm{c}}^{2} \tau_{\mathrm{E}} / 2$

If we now choose the condition of equal initial field strength, i.e. $\bar{E}_{\mathrm{c}}=\left(\bar{E}_{0}\right)_{\mathrm{c}}$, a reasonable comparison requires that $\tau_{\mathrm{E}}=2 t_{\mathrm{p}}$. However, choosing the condition $t_{\mathrm{p}}=\tau_{\mathrm{E}}$, the equality $\left(\bar{E}_{0}\right)_{\mathrm{c}}=\bar{E}_{\mathrm{c}} \times 2^{1 / 2}$ has to be used for the comparison.

\section{A.c. pulses}

For the case of a.c. pulses of angular frequency $\omega=2 \pi \nu$, where $\nu$ is the frequency, and duration $t_{\mathrm{p}}$, the term $[\Delta \phi(E)]^{2}$ in eqn. (17) has to be replaced by the effective peak value $\left\{[\Delta \hat{\phi}(\hat{E})]^{2} / 2\right\} /\left(1+\mathrm{w}^{2} \tau_{\mathrm{p}}^{2}\right)$. At low frequencies $\omega \ll \tau_{\mathrm{p}}^{-1}$, by substitution in eqn. (19), we obtain for a spherical ccll distribution

$\bar{W}_{\mathrm{p}, \mathrm{c}}=G_{\mathrm{p}}\left[\frac{3 \bar{a}}{2} f(\lambda)\right]^{2} \frac{\left(\hat{E}_{\mathrm{c}}\right)^{2}}{2} t_{\mathrm{p}}$

Comparison with data from CD pulses for instance, must be based on the equality

$\left(\hat{\bar{E}}_{\mathrm{c}}\right)^{2} t_{\mathrm{p}}=\bar{E}_{0, \mathrm{c}}^{2} \tau_{\mathrm{E}}$

In any case $W_{\mathrm{p}}$ will reach a limiting value $W_{\mathrm{p}, \mathrm{c}}$, independent of $t_{\mathrm{p}}$, when the electroporated membrane starts to conduct ions appreciably through the pores.

\section{PHYSICAL CHEMISTRY OF DNA ELECTRODELIVERY}

Electroporative DNA transfer is obviously is a multiphase process favoured by adsorption of DNA on the cell surface [31,33,39]. As well as long-lived adsorption of DNA to the cell state $\mathrm{C}$, there are certainly transient short-lived contacts of DNA with cell surface structures such as cell walls and with the plasma membrane. The transport of DNA through the cell wall and the electroporated membrane is certainly also determined by the polyelectrolyte nature of DNA (condensed counter-ions and bound water) and by the cell surface charges.

\section{Cell surface contact of DNA}

In order to describe the interaction of DNA with cell surface in DNA "electrodelivery" we couple the state hysteresis $C \rightleftarrows P$ (scheme (1)) to association- 
dissociation processes of the DNA (D) and to the (electro)diffusion of DNA through the electroporated patches $\mathrm{P}$ into the cell interior [31]. In the scheme $\mathrm{D}+m \mathrm{C} \rightleftharpoons \mathrm{D} \cdot \mathrm{C}$<smiles>C=C</smiles>

$\mathrm{D}+m \mathrm{P} \rightleftharpoons \mathrm{D} \cdot \mathrm{P} \longleftrightarrow \mathrm{P} \cdot \mathrm{D} \longrightarrow \mathrm{D}_{\text {in }} \longrightarrow \mathrm{TC}$

the states $\mathrm{D} \cdot \mathrm{C}$ and $\mathrm{D} \cdot \mathrm{P}$ denote the surface-bound $\mathrm{DNA}, \mathrm{D} \cdot \mathrm{P} \leftrightarrow \mathrm{P} \cdot \mathrm{D}$ represents the transmembrane diffusion of DNA, $D_{\text {in }}$ is the DNA that entered the cytoplasma (transfection), TC is the final state of the transformed cell and $m$ is the maximum number of surface (binding) sites for DNA per cell.

The data analysis can now be based on the assumption that the transformation probability increases with the increase in the concentration of the transient contact state $\mathrm{D} \cdot \mathrm{P}$. In the regime where we can assume that the concentration [TC] of transfected cells is given by the proportionality $[\mathrm{TC}] \sim[\mathrm{D} \cdot \mathrm{P}]$, the degree of transfection $\xi_{T}$ is given by

$\xi_{\mathrm{T}}=\frac{T}{T_{\max }}=\frac{[\mathrm{TC}]}{[\mathrm{TC}]_{\max }}=\frac{[\mathrm{D} \cdot \mathrm{P}]}{[\mathrm{D} \cdot \mathrm{P}]_{\max }}$

where $T$ is the number of transfected cells and $T_{\max }$ is the maximum value.

If we apply mass action laws to the stationary states of DNA binding to $m$ independent membrane sites, we obtain for scheme (30)

$\frac{[\mathrm{D} \cdot \mathrm{P}]}{[\mathrm{D} \cdot \mathrm{P}]_{\max }}=\frac{[\mathrm{D}]}{[\mathrm{D}]+\bar{Q}(\mathrm{D})}=\frac{m[\mathrm{C}]}{m[\mathrm{C}]+\bar{Q}(\mathrm{C})}$

In eqn. (32), the distribution constants $\bar{Q}(\mathrm{D})$, in terms of DNA concentration, and $\bar{Q}(\mathrm{C})$, in terms of cell surface sites, should be the same. Thus $\bar{Q}(\mathrm{D})=\bar{Q}(\mathrm{C})=\bar{Q}$. Hence

$$
\begin{aligned}
\bar{Q} & =[\mathrm{D}] \frac{m[\mathrm{C}]+m[\mathrm{P}]}{[\mathrm{D} \cdot \mathrm{C}]+[\mathrm{D} \cdot \mathrm{P}]} \\
& =K_{1} \frac{1+K_{0}}{1+K_{0}^{\prime}}
\end{aligned}
$$

where the individual distribution constants are defined by $K_{0}=[\mathrm{P}] /[\mathrm{C}], K_{1}=$ [D] $m[\mathrm{C}] /[\mathrm{D} \cdot \mathrm{C}], K_{0}^{\prime}=[\mathrm{D} \cdot \mathrm{P}] /[\mathrm{D} \cdot \mathrm{C}]$ and $K_{2}=K_{1} K_{0} / K_{0}^{\prime}$.

Now eqns. (31) and (32) are combined and reorganized to yield the practically useful expression

$$
\frac{1}{T}=\frac{1}{T_{\max }}\left(1+Q \frac{1}{[\mathrm{D}]}\right)
$$

For graphical data evaluation it should be noted that [D] in eqn. (34) refers to free unbound DNA. However, if $T^{-1}$ is plotted versus $\left[\mathrm{D}_{\mathrm{T}}\right]^{-1}$, where $\left[\mathrm{D}_{\mathrm{T}}\right]$ is the total 


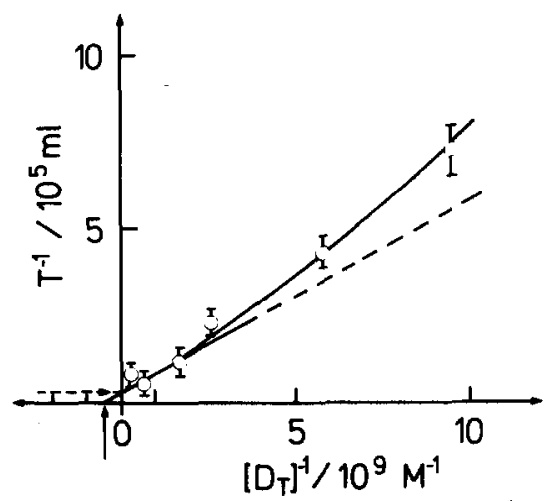

Fig. 7. Electrotransformation of intact C.glut. cells at a cell density of $10^{10} / \mathrm{ml}$ at $4^{\circ} \mathrm{C}$ : quasi-rectangular pulse ( $E_{0}=10 \mathrm{kV} \mathrm{cm}{ }^{-1}, t_{\mathrm{p}}=5 \mathrm{~ms}$ ); medium; $0.272 \mathrm{M}$ sucrose, $0.01 \mathrm{M}$ Hepes buffer, $1 \mathrm{mM} \mathrm{MgCl}_{2}$, $\mathrm{KOH}$ adjusted to yield pH 7.4 at $4^{\circ} \mathrm{C}$, pUL-330-plasmid DNA (5.2 kb). The "Langmuir adsorption type" relationship between the number $T$ of transformants per millilitre and the total concentration $\left[D_{\mathrm{T}}\right]$ of DNA is represented in terms of eqn. (34). The broken line refers to the linear dependence on $[D]^{-1}$; we use $M($ DNA $)=3.43 \times 10^{6} \mathrm{~g} / \mathrm{mol}$.

concentration of the DNA applied, the parameters $T_{\max }$ and $Q$ can readily be estimated from the range of high $\left[\mathrm{D}_{\mathrm{T}}\right]$ values or low $\left[\mathrm{D}_{\mathrm{T}}\right]^{-1}$ values respectively. For instance, the transformation of Corynebacterium glutamicum (C.glut.) is dependent on the concentrations of DNA and cells [20]. Data evaluation according to eqn. (34) is shown in Fig. 7 [31]. We obtain $\bar{Q}=(2 \pm 1) \times 10^{-9} \mathrm{M}$ (DNA), $m=25$ and $T_{\max }=3.3 \times 10^{5} \mathrm{ml}^{-1}$ at a cell density of $10^{10} \mathrm{cell} / \mathrm{ml}$ for quasi-rectangular pulses $\left(E_{0}=10.5 \mathrm{kV} \mathrm{cm}^{-1}, t_{\mathrm{p}}=5 \mathrm{~ms}\right)$.

\section{Orientation of DNA and bacterium rods}

Since DNA is a linear polyelectrolyte, an applied electric field $E$ displaces the counter-ion atmosphere relative to the polyanionic DNA rod, orienting the induced dipolar rod with its long axis in the direction of $E$ [43]. Figure 8 shows how counter-ion flow along the DNA induces a stationary flow dipole which orients.

A superhelical plasmid DNA can also be considered as an overall rod which in an applied electric field is oriented. The rotation time constant is dependent on $E$ and on the length of the rotation axis. For the plasmid pPBRN $3(2 \mathrm{~kb})$ the orientation time constant is about $5 \mu \mathrm{s}$ at $E=20 \mathrm{kV} \mathrm{cm}-1, \Delta t=10 \mu \mathrm{s}$ and $293 \mathrm{~K}$ [44]. If the pulse duration is in the millisecond time range, the DNA orientation can usually be considered as very rapid. However, at low ionic content of the solution, pDNA may partially uncoil [44].

Cell surface structures such as a cell wall and the outer side of the plasma membrane are usually net negatively charged and therefore surrounded by a net positively charged counter-ion atmosphere. Rod-like cells like bacteria are simi- 


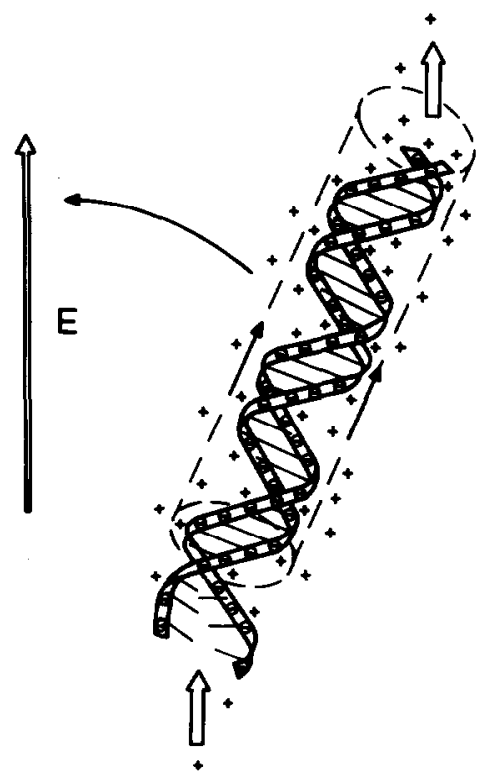

Fig. 8. Ionic atmosphere flow polarization of DNA induced by counter-ion displacement along the helix axis in an external electric field $\boldsymbol{E}$. The stationary induced dipole moment is due to counter-ion deficiency at the unscreened phosphate groups of the lower end and to counter-ion accumulation at the upper end of double helix.

larly flow polarized as DNA and orient with the long axis in the direction of the external field (Fig. 9), provided that the pulse duration $t_{\mathrm{p}}$ is large enough compared with the rotational time constant, which is estimated to be of the order of milliseconds for bacteria like E.coli with $L \approx 3 \mu \mathrm{m}$ in fields of the order of several $\mathrm{kV} \mathrm{cm}^{-1}$ [44].

\section{Electrodiffusion of DNA and cells}

Cell surfaces at $\mathrm{pH} 7$ are normally polyanionic. Elongated cells like bacteria and DNA not only orient their long axes in the direction of the external field, but as polyanions they move electrophoretically in a drift direction opposite to the direction of the external field vector (Fig. 9).

Generally, the velocity vector $\boldsymbol{v}_{i}$ of a particle $i$ is related to the field vector $\boldsymbol{E}$ through

$\boldsymbol{v}_{i}=\left(z_{i} /\left|z_{i}\right|\right) u_{i} \boldsymbol{E}$

where $z_{i}$ is the charge number (with sign) and $u_{i}$ is the electric mobility of the 


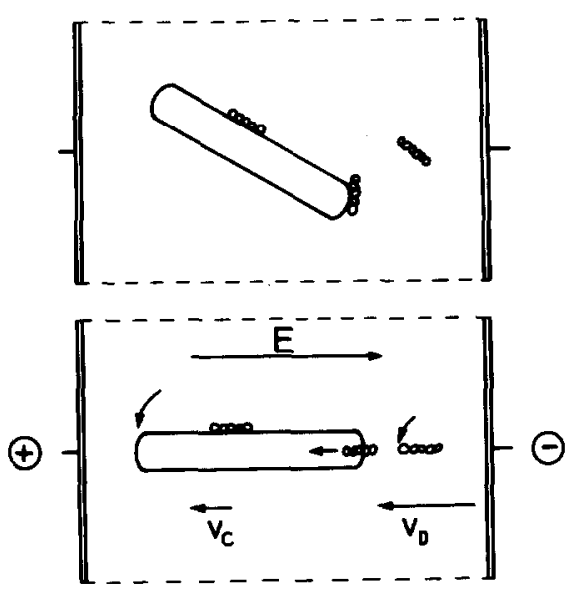

Fig. 9. Model for the interaction of superhelical plasmid DNA and bacterium rods in an applied electric field $E$. Upper part: $E=0$; some DNA is adsorbed to the bacterium surface. At large fields there can be complete orientation of the long axes of the particles in the direction of $E$ and electrophoretic movement of the anionic particles. Since $v(D)>v(C)$, DNA can presumably be electrophoretically drawn through one of the electroporated pole caps of the bacterium rod.

particle $i$. For anionic particles, $z_{i} /\left|z_{i}\right|=-1$. Therefore the electrophoretic movements of DNA D and bacterium cells $C$ arc described by

$v(\mathrm{D})=-u(\mathrm{D}) \mathrm{E}$

$v(\mathrm{C})=-u(\mathrm{C}) E$

respectively.

It is known that the DNA mobility $u(D) \approx(1.5 \pm 0.5) \times 10^{-4} \mathrm{~cm}^{2} \mathrm{~V}^{-1} \mathrm{~s}^{-1}$ [44] is independent of the chain length. Generally, the electric mobility tensor $u_{i}$ of (geometrically anisotropic) particles is dependent on the surface charge density and the particle size (and on the direction of the longest particle axis relative to $E$ ). Cell surface structures are usually less charged than DNA and cells are larger than DNA; therefore $u(D)>u(C)$. As a consequence, in the presence of a field $E$, DNA moves faster than larger cells (Fig. 9). Hence it is conceivable that DNA is electrophoretically inserted in, and drawn through, the electroporated membrane cap facing the cathodic electrode of the measuring chamber.

Electroporative bacterium transformation requires a long pulse duration. The reason is most probably the network structure of the porous cell wall. Externally applied DNA first has to migrate (electro)diffusively through the wall structures (equivalent to a higher solution viscosity) before entering the plasma membrane.

Finally, it should be realized that the transformed cells $\mathrm{T}$ or the degree of transformation $\xi_{\mathrm{T}}=T / T_{\max }$ reflects the last member of the sequence

$E \rightarrow \Delta \phi(E) \rightarrow \Delta \xi_{\mathrm{p}} \rightarrow \Delta \xi_{\mathrm{T}}$ 
Therefore $\xi_{\mathrm{T}}$ is likely to depend on $E$ and $t_{\mathrm{p}}$ via the degree of poration $\xi_{\mathrm{p}}$ in a complicated manner. Nevertheless, parameters related to $\xi_{\mathrm{T}}$ and $\xi_{\mathrm{p}}$, and discussed in this paper, are useful with respect to studies of the mechanisms of membrane electroporation and gene electrodelivery.

\section{ACKNOWLEDGEMENTS}

I thank my co-workers Dr. E. Boldt, Dr. H. Wolf and Dr. E. Werner for providing unpublished results and Mrs. M. Pohlmann for careful typing of the manuscript. The financial support of the Deutsche Forschungsgemeinschaft (Grant $\mathrm{Ne} 227$ ) and the Fonds der Chemie is gratefully acknowledged.

\section{REFERENCES}

1 E. Neumann, A.E. Sowers and C.A. Jordan (Eds.), Electroporation and Electrofusion in Cell Biology, Plenum Press, New York, 1989.

2 A.E. Sowers (Ed.), Cell Fusion, Plenum Press, New York, 1987.

3 D.C. Chang, B.M. Chassy, J.A. Saunders and A.E. Sowers (Eds.), Guide to Electroporation and Electrofusion, Academic Press, San Diego, CA, 1992.

4 E. Neumann and K. Rosenheck, J. Membr. Biol., 10 (1972) 279.

5 E. Neumann and K. Rosenheck, J. Membr. Biol., 14 (1973) 194.

6 U. Zimmermann, J. Schulz and G. Pilwat, Biophys. J., 13 (1973) 1005.

7 U. Zimmermann, G. Pilwat and F. Riemann, Z. Naturforsch., 29c (1974) 394.

8 U. Zimmermann, F. Riemann and G. Pilwat, Biochim. Biophys. Acta, 436 (1976) 460.

9 H. Berg, W. Förster, H.-E. Jacob, W. Jungstand and P. Mühlig, Stud. Biophys., 74 (1978) 31.

10 M. Senda, J. Takeda, A. Shunnosuke and T. Nakamura, Plant Cell Physiol., 20(7) (1979) 1441.

11 E. Neumann, G. Gerisch and K. Opatz, Naturwissenschaften, 67 (1980) 414.

12 H. Weber, W. Förster, H.-E. Jacob and H. Berg in G.G. Stewart and I. Russel (Eds.), Current Developments in Yeast Research. Advances in Biotechnology, Pergamon Press, Toronto, 1981, p. 219.

13 U. Zimmermann and P. Scheurich, Planta, 151 (1981) 26.

14 J. Teissié, V.P. Knutson, T.Y. Tsong and M.D. Lane, Science, 216 (1982) 537.

15 Y. Mouneimne, P.F. Tosi, Y. Gazitt and C. Nicolau, Biochem. Biophys. Res. Commun., 159 (1989) 34.

16 E. Neumann, M. Schaefer-Ridder, Y. Wang and P.H. Hofschneider, EMBO J., 1 (1982) 841.

17 R.A. Winegar, J.W. Phillips, J.H. Youngblom and W.F. Morgan, Mutat. Res., 225 (1989) 49.

18 H. Hashimoto, H. Morikawa, Y. Yamada and A. Kimura, Appl. Microbiol. Biotechnol., 21 (1985) 336.

19 W.J. Dower, J.F. Miller and C.W. Ragsdale, Nucleic Acids Res., 16(13) (1988) 6127.

$20 \mathrm{H}$. Wolf, A. Pühler and E. Neumann, Appl. Microbiol. Biotechnol., 30 (1989) 283.

21 B.M. Chassy, A. Mercenier and J. Flickinger, TIBTECH, 6 (1988) 303.

22 W. Förster and E. Neumann, in E. Neumann, A.E. Sowers and C.A. Jordan (Eds.), Electroporation and Electrofusion in Cell Biology, Plenum Press, New York, 1989, p. 299.

23 Bio-Rad Laboratories, Bulletins, Richmond, CA, 1988.

24 E. Neumann, in E. Neumann, A.E. Sowers and C.A. Jordan (Eds.), Electroporation and Electrofusion in Cell Biology, Plenum Press, New York, 1989, p. 61.

25 E. Neumann, Ferroelectrics, 86 (1988) 325.

26 D.C. Chang, and T.S. Reese, Biophys. J., 58 (1990) 1.

27 I.G. Abidor, V.B. Arakelyan, L.V. Chernomordik, Y.A. Chizmadzhev, V.F. Pastushenko and M.R. Tarascvich, Bioelcctrochcm. Biocncrg., 6 (1979) 37. 
8 L.V. Chernomordik and Y.A. Chizmadzhev, in E. Neumann, A.E. Sowers and C.A. Jordan (Eds.), Electroporation and Electrofusion in Cell Biology, Plenum Press, New York, 1989, p. 83.

9 J.D. Litster, Phys. Lett., 53A (1975) 193.

0 J.C. Weaver and K.T. Powell, in E. Neumann, A.E. Sowers and C.A. Jordan (Eds.), Electroporation and Electrofusion in Cell Biology, Plenum Press, New York, 1989, p. 111.

1 E. Neumann, A. Sprafke, E. Boldt and H. Wolf, in D.C. Chang, B.M. Chassy, J.A. Saunders and A.E. Sowers (Eds.), Guide to Electroporation and Electrofusion, Academic Press, San Diego, CA, 1991 , p. 77.

2 E. Neumann and E. Boldt, in M.J. Allen, S.F. Cleary and F.M. Hawkridge (Eds.), Charge and Field Effects in Biosystems-2, Plenum Press, New York, 1989, p. 373.

3 E. Neumann and E. Boldt, in C. Nicolau and D. Chapman (Eds.), Horizons in Membrane Biotechnology, Progress in Clinical and Biological Research, Wiley-Liss, New York, 1990, p. 69.

4 T.K. Wong and E. Neumann, Biochem. Biophys. Res. Commun., 107 (1982) 584.

5 B.R. Jennings and V.J. Morris, J. Colloid Interface Sci., 50 (1975) 352.

6 F. Pliquett, Z. Biol., 116 (1967) 9.

7 W. Harbich and W. Helfrich, Z. Naturforsch., 34a (1979) 1063.

3 H. Itoh, M. Hibino, M. Shigemori, M. Koishi, A. Takahashi, T. Hayakawa and K. Konosita, Jr., Proc. SPIE, 1204 (1990) 49.

7 T.-D. Xie, L. Sun and T.Y. Tsong, Biophys. J., 58 (1990) 13.

J H.P. Schwan, Adv. Biol. Med. Phys., 5 (1957) 147.

1 H. Fricke, J. Appl. Phys., 24 (1953) 644.

2 A.J.H. Sale and W.A. Hamilton, Biochim. Biophys. Acta, 163 (1968) 37.

3 M. Eigen and G. Schwarz, in B. Pesce (Ed.), Electrolytes, Pergamon, Oxford, 1962, p. 309.

7 E. Werner, Ph.D. Thesis, University of Bielefeld, 1989. 